\title{
A New Pharmacokinetic Model of Propofol for Japanese Patients
}

\author{
Masahito Omote, Shunsuke Tachibana, Yasuyuki Tokinaga* (1), Michiaki Yamakage \\ Department of Anesthesiology, Sapporo Medical University School of Medicine, Sapporo, Japan \\ Email: *y.tokinaga @sapmed.ac.jp
}

How to cite this paper: Omote, M., Tachibana, S., Tokinaga, Y. and Yamakage, M. (2019) A New Pharmacokinetic Model of Propofol for Japanese Patients. Open Journal of Anesthesiology, 9, 179-188. https://doi.org/10.4236/ojanes.2019.99017

Received: August 25, 2019

Accepted: September 16, 2019

Published: September 19, 2019

Copyright () 2019 by author(s) and Scientific Research Publishing Inc. This work is licensed under the Creative Commons Attribution International License (CC BY 4.0).

http://creativecommons.org/licenses/by/4.0/ (c) (i) Open Access

\begin{abstract}
Target-controlled infusion (TCI) of propofol is used for general anesthesia. However, the only pharmacokinetic parameter commercially used for Japanese patients is weight, and pharmacokinetic models are based on European physical attributes. Drug metabolism also differs in races. This study aimed to identify optimal continuous doses of propofol for Japanese patients and to create a simulated pharmacokinetic (PK) model. Thirty Japanese patients were enrolled. Patients received a constant infusion of $9 \mathrm{mg} / \mathrm{kg} / \mathrm{h}$ of propofol. Arterial blood samples were collected and the time course of plasma propofol concentrations was modeled using the nonlinear mixed effects model (NONMEM) three-compartmental PK model. We validated the model by intravenously injecting 10 patients with a TCI driver system programmed with the NONMEM model. Our model's performance was evaluated using the median prediction error (MDPE), median absolute prediction error (MDAPE), and Wobble. We analyzed 320 blood samples for model building and 160 samples for validating our new model. The calculated parameters for the three-compartmental PK model were volume [V1, 3.58; V2, $13.0+0.49 \times$ (Age-64); and V3, 186] and elimination clearance [CL1, $0.77+(\mathrm{WT}-54) \times$ $0.04+(\mathrm{HT}-158) \times 0.03 ; \mathrm{CL} 2,0.89+0.12 \times($ Age-64); and CL3, $0.98 \times \exp$ ((Age-64)/10)]. The new model improved MDPE, MDAPE, and Wobble values $(11.5 \% \pm 43.8 \%, 14.3 \% \pm 33.0 \%$, and $25.0 \% \pm 21.3 \%$, respectively). We created a new pharmacokinetic model for Japanese patients, which is more accurate than the three existing models applied to Japanese populations. Electronic document is a "live" template. The various components of your paper [title, text, heads, etc.] are already defined on the style sheet, as illustrated by the portions given in this document.
\end{abstract}

\section{Keywords}

Propofol, Pharmacokinetics, Target-Controlled Infusion, Japanese 


\section{Introduction}

Target-controlled infusion (TCI) of propofol is used worldwide to induce and maintain general anesthesia. However, the pharmacokinetic parameters of TCI devices for propofol that are commercially used in Japan include only the patient's weight. Various pharmacokinetic parameters have been published; however, these models are based on European physical attributes, which differ considerably with regard to fat and muscle composition from those of Japanese populations [1] [2]. In addition, drug metabolism differs in races [3]. Using only a European standard of weight may cause bias in the use of a TCI device, which could potentially lead to overdoses in Japanese patients. Furthermore, this bias is likely remarkable, particularly when TCI devices are used for obese patients. Tachibana et al. [4] reported that the body mass index (BMI) influences blood propofol concentrations, and may lead to a propofol overdose in obese patients when assessing propofol concentrations by using the Marsh model. Lampotang et al. [5] reported that the plasma and effect-site concentrations of propofol predicted using the Marsh model to achieve loss of consciousness vary among Caucasians, Chinese, Africans, and Indians. The present study aimed to identify optimal continuous doses of propofol for Japanese patients and create a simulated PK model using NONMEM version 7.2 software (ICON Clinical Research, LLC, North Wales, PA, USA) [6].

\section{Methods}

The protocol of this study was designed in accordance with the principles outlined in the Declaration of Helsinki. The Ethics Committee of Sapporo Medical University Hospitals (Sapporo, Japan) approved the study (approval No. 272-3) on March 14, 2015. Written informed consent was obtained from all patients. The study was registered at University Hospital Medical Information Network Clinical Trials Registry (registry No. UMIN000029932).

\subsection{Recruitment of Patients}

This was a prospective observational study. Thirty patients living in Japan from birth were enrolled in this study. All patients were scheduled for elective brain, head, or neck surgery under general anesthesia at Sapporo Medical University Hospital. The exclusion criteria were as follows: age $<20$ years or age $>70$ years; American Society of Anesthesiologists (ASA) physical status of 3 to 5; allergic to propofol; a daily intake of psychoactive drugs; known or suspected drug or alcohol abuse; and cardiac, hepatic, renal, or neurological impairment; extremely obese or lean body.

\subsection{Creating a Pharmacokinetic Model for Propofol}

We planned to obtain blood samples from 20 patients to create a pharmacokinetic model of propofol. The patients were not premedicated before arriving at the operating room where cardiac parameters were monitored with electrocar- 
diography and brain parameters were monitored with the bispectral index (BIS) (VISTA; Covidien, Dublin, Ireland). Blood pressure, oxygen saturation, and end-tidal carbon dioxide concentrations were also monitored. A 20-gauge cannula was inserted into a forearm vein, and a 22 -gauge radial artery catheter was also inserted for arterial blood sampling and invasive blood pressure monitoring.

All enrolled patients received approximately $3 \mathrm{~mL} / \mathrm{kg} / \mathrm{h}$ of crystalloid fluid during the study period. Before inducing anesthesia, all patients maintained spontaneous ventilation via a facemask delivering $100 \%$ oxygen. Fentanyl (Daiichi-Sankyo Pharmaceuticals, Tokyo, Japan) was injected at a dose of $2 \mu \mathrm{g} / \mathrm{kg}$ and propofol (1\% Diprivan; AstraZeneca, Cambridge, UK) was infused at a constant rate of $9 \mathrm{mg} / \mathrm{kg} / \mathrm{h}$ (based on the patient's actual body weight) for at least 2 hours. All patients were mechanically ventilated under endotracheal intubation. Anesthesia was maintained with propofol and remifentanil. Arterial blood samples were collected at $1 \mathrm{~min}, 2 \mathrm{~min}, 4 \mathrm{~min}, 8 \mathrm{~min}, 12 \mathrm{~min}, 20 \mathrm{~min}, 30 \mathrm{~min}, 40 \mathrm{~min}, 50$ min, $60 \mathrm{~min}, 75 \mathrm{~min}, 90 \mathrm{~min}$, and $120 \mathrm{~min}$ after starting anesthesia; and then collected at the emergence from anesthesia and at the completion of general anesthesia. The blood samples were immediately centrifuged to obtain the plasma fraction. The plasma was stored at $-80^{\circ} \mathrm{C}$ until propofol analysis.

\subsection{Propofol Assays}

Propofol concentrations were measured using a high-performance liquid chromatography (HPLC) system with a fluorescence detector (EICOM, Kyoto, Japan). To confirm the propofol concentration, we used previously described HPLC methods as the basis for the studies we conducted [7].

In brief, $200 \mu \mathrm{L}$ of a blood plasma sample was mixed with $200 \mu \mathrm{L}$ of methanol containing $10 \mu \mathrm{L}$ of 2-tert-butyl-6-methylphenol $(0.1 \mu \mathrm{L} / \mathrm{mL})$ as the internal standard. After mixing, the samples were centrifuged for $10 \mathrm{~min}$ at $15,000 \times \mathrm{g}$. The supernatant was filtered and injected into the high-performance liquid chromatograph for analysis. The propofol concentration was calculated, based on the ratio of the propofol and peaks in the HPLC chromatogram.

The mobile phase comprised $0.1 \mathrm{M}$ phosphate buffer and methanol (3:7). The flow rate was $0.5 \mathrm{~mL} / \mathrm{min}$, and the column temperature was $25^{\circ} \mathrm{C}$. The fluorescence detector was set at an excitation of $270 \mathrm{~nm}$ and an emission of $310 \mathrm{~nm}$. Drug separation was achieved with a C18 column $(4.6 \mathrm{~mm} \times 30 \mathrm{~mm}$; PP-ODS II; EICOM), which was maintained at $25^{\circ} \mathrm{C}$.

\subsection{Comparison with Published Pharmacokinetic Models}

The Marsh model [8], Schnider model [9], and General Purpose model [10] for patient simulation were compared using TIVA Trainer software (Gutta B.V., Aerdenhout, Netherlands). The simulation program calculated the predicted blood propofol concentrations $\left(C p_{\text {pred }}\right)$. At each time point, the performance error was calculated with measured blood concentrations ( $\left.C p_{\text {measured }}\right)$ as

$$
P E \%=\left(C p_{\text {measured }}-C p_{\text {pred }}\right) / C p_{\text {pred }} \times 100,
$$


in which PE\% is the percentage prediction error and $C p_{\text {pred }}$ is the blood propofol concentration predicted by a pharmacokinetic model. The $\mathrm{d}$ (i.e., a measure of bias), the MDAPE (i.e., a measure of inaccuracy), and Wobble (i.e., the variation in performance error around the MDPE) were determined for each patient. The MDPE, MDAPE, and Wobble were calculated to measure bias at each time point for each individual (i), having Ni blood samples, as follows: MDPEi $=$ Median $[$ PEij, $\mathrm{j}=1, \cdots, \mathrm{Ni}]$; MDAPEi $=$ Median $[$ PEij, $\mathrm{j}=1, \cdots, \mathrm{Ni}]$ and Wobblei $=$ Mean [PEij-MDPEij, $j=1, \cdots, \mathrm{Ni}$ ]. The predicted performance was calculated using $\mathrm{R}$ language, version 3.4.2 (R Foundation for Statistical Computing, Vienna, Austria).

\subsection{Model Building}

After comparison with the published model, the new parameters were calculated to obtain better MDPE, MDAPE and Wobble values. A three-compartment model was used for defining the basic structural PK model because of referred to the Marsh model, Schnider model, and General Purpose model. Interindividual variability was assumed and tested. We modeled the time course of propofol plasma concentrations by using a three-compartmental PK model with central volume (V1), rapid peripheral volume (V2), and slow peripheral volume (V3), metabolic clearance (CL1), rapid peripheral clearance (CL2); and slow peripheral clearance (CL3). The models were estimated and evaluated using NONMEM version 7.2 (ICON Clinical Research, LLC). By using NONMEM software, the measured plasma concentration data were analyzed, and the population pharmacokinetic parameters of propofol were fitted by using Wings for NONMEM software. Age, height, and body weight were possible covariates for the model parameters. We used these parameters as covariates for the PK model because of its usefulness of expression for the patient's body shape and easiness to getting patient's preoperative information.

\subsection{Model Validation}

We validated our model, as follows. A TCI driver programmed with our new model intravenously injected 10 patients with propofol to achieve a blood concentration (Cp) of $3.0 \mu \mathrm{g} / \mathrm{mL}$ and collected arterial blood samples at the time points described previously. To administer propofol, we used our original program. A syringe pump was connected via RS232C terminal to the computer.

The performance of the model in each patient was assessed using the MDPE, MDAPE, and Wobble. Blood pressure degradation after achieving a $\mathrm{Cp}$ of 3.0 $\mu \mathrm{g} / \mathrm{mL}$ was measured, and adverse effects such as vascular pain and hypotension were evaluated as the secondary outcomes.

\section{Results and Discussions}

Table 1 lists the demographics and the mean time required for the patients to return to consciousness. The 2017 National Health and Nutrition Survey, which 
Table 1. Patients' demographic data.

\begin{tabular}{cc}
\hline Age $(\mathrm{y})$ & $61 \pm 25$ \\
\hline Sex $(\mathrm{M} / \mathrm{F})$ & $9 / 11$ \\
Weight $(\mathrm{kg})$ & $57 \pm 14$ \\
Height $(\mathrm{cm})$ & $163 \pm 13$ \\
Body mass index $\left(\mathrm{kg} / \mathrm{m}^{2}\right)$ & $21.9 \pm 5.3$ \\
\hline
\end{tabular}

Data are presented as the mean \pm the standard deviation or as the number. All patients living in Japan from birth were scheduled for elective brain, head, or neck surgery under general anesthesia at Sapporo Medical University Hospital. Their American Society of Anesthesiologists physical status was 1 to 2 . The exclusion criteria were as follows: age $<20$ years or age $>70$ years; American Society of Anesthesiologists (ASA) physical status of 3 to 5; allergic to propofol; a daily intake of psychoactive drugs; known or suspected drug or alcohol abuse; and cardiac, hepatic, renal, or neurological impairment; extremely obese or lean body. $F$. female, $M$ : male.

was administered by the Japanese Ministry of Health, Labour and Welfare (Tokyo, Japan) [11], reported that the mean BMI in male and female individuals were $23.8 \mathrm{~kg} / \mathrm{m}^{2}$ and $22.6 \mathrm{~kg} / \mathrm{m}^{2}$, respectively. Blood loss was less than $100 \mathrm{~mL}$ for each patient during the study. The patients were of medium build. Three hundred twenty blood samples were analyzed for model building and 160 blood samples were analyzed for model validation. No adverse events such as hypotension occurred in this study.

Figure 1 shows the comparison of our model with the Marsh, Schnider, and General Purpose models. Table 2 shows the pharmacokinetic performance of these models. Most blood propofol concentrations during the phase of maintained anesthesia were underestimated when propofol was infused at a constant rate. The General Purpose model had the worst performance among the three models, despite having been the basis for nearly all published calculations.

We revised the model by including covariates for age, height, and body weight to optimize the relationship between the observed data, predicted data, and pharmacokinetic data. Table 3 shows that the pharmacokinetic data derived from the new model and the Schnider model were similar. Age was a significant covariate for rapid peripheral volume and rapid peripheral clearance, whereas height and body weight were significant covariates for metabolic clearance.

We then evaluated this new model using a TCI driver programmed with the new model to achieve a $\mathrm{Cp}$ of $3.0 \mu \mathrm{g} / \mathrm{mL}$. Table 4 shows the patient demographics, average elapsed time to consciousness, and blood pressure degradation. Figure 2 shows the percentage prediction error for the new model and Table 5 shows the pharmacokinetic performance of intravenously injected propofol. General anesthesia was induced within $14.0 \pm 4.3 \mathrm{~min}$. During validation, no patient complained of pain at the injection site in the arm or experienced hypotension. The MDPE and MDAPE were $<30 \%$, which indicated the new model had good performance.

Many studies have used the pharmacokinetic parameters established by Marsh et al. [8], and have assessed these parameters in obese, pediatric, and elderly 

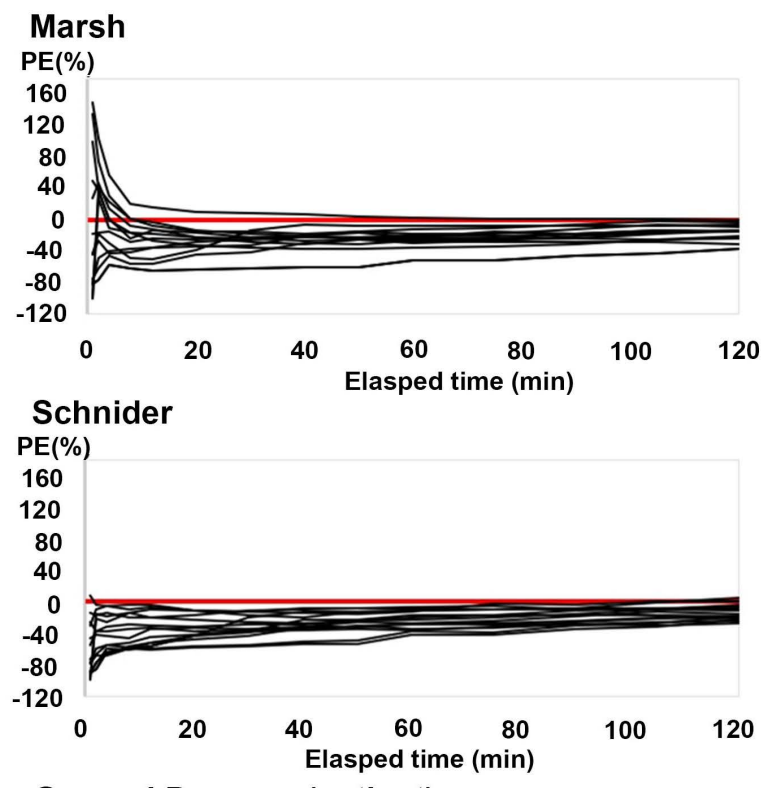

General Purpose (patient)

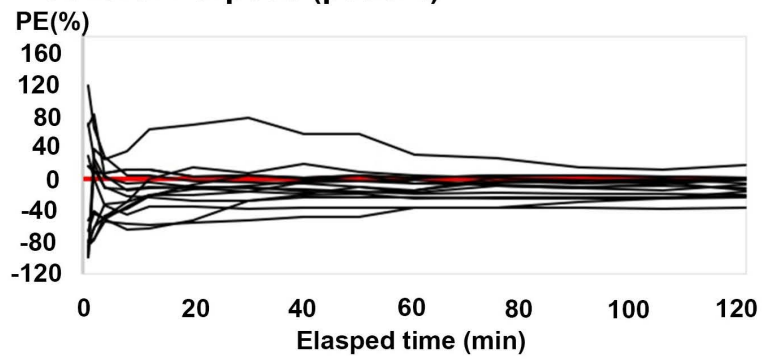

Figure 1. The percent prediction error (PE\%) for the Marsh model, Schnider model and General Purpose model.

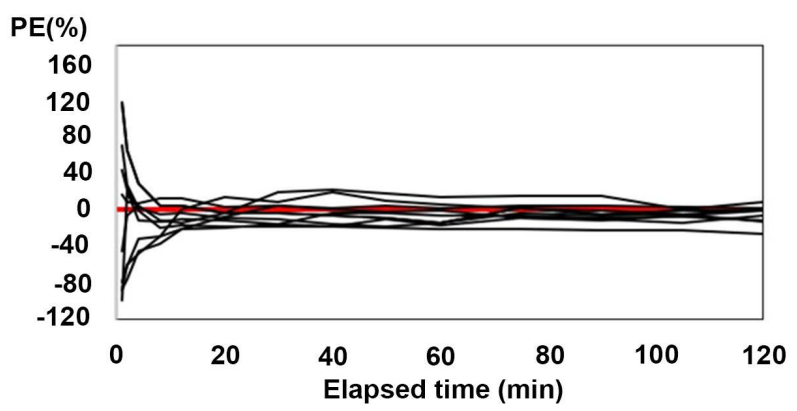

Figure 2. The percent prediction error (PE\%) for the new model of target-controlled infusion at $3 \mu \mathrm{g} / \mathrm{mL}$.

Table 2. Pharmacokinetic performance of the Marsh model, Schnider model, and General Purpose model.

\begin{tabular}{cccc}
\hline Model & MDPE (\%) & MDAPE (\%) & Wobble (\%) \\
\hline Marsh & $-24.5 \pm 35.0$ & $26.8 \pm 30.0$ & $16.1 \pm 15.5$ \\
Schnider & $-27.9 \pm 30.0$ & $28.0 \pm 29.7$ & $11.9 \pm 12.6$ \\
General Purpose (patient) & $-40.3 \pm 30.0$ & $40.7 \pm 28.9$ & $11.3 \pm 6.9$ \\
\hline
\end{tabular}

The data are presented as the mean \pm the standard deviation. MDAPE: median absolute prediction error, $M D P E$ : median prediction error. 
Table 3. Pharmacokinetic parameters of the new model.

\begin{tabular}{cc}
\hline Volume (L) & \\
\hline V1 & 3.58 \\
V2 & $13.0+0.49 \times($ Age -64$)$ \\
V3 & 186 \\
\hline Clearances $(\mathrm{L} / \mathrm{min})$ & $0.77+(\mathrm{WT}-54) \times 0.04+(\mathrm{HT}-158) \times 0.03$ \\
CL1 & $0.89+0.12 \times($ Age -64$)$ \\
CL2 & $0.98 \times \exp (($ Age -64$) / 10)$ \\
CL3
\end{tabular}

$A g e$, age (in years), $C L 1$ : metabolic clearance. $C L 2$ : rapid peripheral, $C L 3$ : slow peripheral, $H T$ : height $(\mathrm{cm})$; V: central volume, V2: rapid peripheral volume, V3: slow peripheral volume, WT: body weight $(\mathrm{kg})$.

Table 4. Patients' demographic data and validation statistics.

\begin{tabular}{cc}
\hline Age $(\mathrm{y})$ & $54 \pm 13$ \\
\hline Sex $(\mathrm{M} / \mathrm{F})$ & $5 / 5$ \\
Weight $(\mathrm{kg})$ & $61 \pm 27$ \\
Height $(\mathrm{cm})$ & $161 \pm 11$ \\
Body mass index $\left(\mathrm{kg} / \mathrm{m}^{2}\right)$ & $21.4 \pm 4.8$ \\
Average elapsed time to consciousness $(\mathrm{min})$ & $4.3 \pm 1.5$ \\
Rate of MAP change after achieving Cp 3.0 $\mu \mathrm{g} / \mathrm{mL}(\%)$ & $16.2 \pm 3.9$ \\
Patients with vascular pain $(\mathrm{n})$ & $0 / 10$ \\
\hline
\end{tabular}

The data are presented as the mean \pm the standard deviation or as the number. $C p$ : predicted blood propofol concentration, $F$ : female, $M$ : male, $M A P$ : mean arterial pressure.

Table 5. Pharmacokinetic performance of the new model.

\begin{tabular}{ccc}
\hline MDPE (\%) & MDAPE (\%) & Wobble (\%) \\
\hline $11.5 \pm 43.8$ & $14.3 \pm 33.0$ & $25.0 \pm 21.3$ \\
\hline
\end{tabular}

The data are presented as the mean percentage \pm the standard deviation. MDAPE: median absolute prediction error, $M D P E$ : median prediction error.

patients. These studies demonstrated that the parameters were clinically acceptable. Li et al. [12] reported that TCI systems using the Marsh model are clinically acceptable for upper abdominal surgery.

Some investigators have found that other models are preferable to the Marsh model. Masui et al. [13] recommend the Schnider model rather than the Marsh model. Rigouzzo et al. [14] report that the Schnider model is more useful for children. Glen et al. [15] recommend the White model rather than the Marsh model. However, our results indicated that the Marsh model performed better than the Schnider model.

The performance of the General Purpose model was the poorest among the three models in the present study, despite its application in most published data. An MDPE less than 15\% and an MDAPE less than 30\% indicate that a TCI sys- 
tem has a clinically acceptable performance [16]. We took into consideration that the pharmacokinetic parameters may have been biased in published studies because most of their data are based on European physical attributes.

Many factors such as cardiac output and hepatic blood flow influence blood propofol concentrations. In addition, weight and body height, which are affected by ethnic differences, can introduce bias. Lampotang et al. [5] reported that propofol concentrations predicted by the Marsh model varied among ethnicities, and created a pharmacodynamic model of propofol-induced loss of consciousness. Our model outperformed the other three models when applied to a Japanese population and may be useful. Moreover, the costs will be reduced because unnecessary drug administration may be cut when our model is used.

Sex may also affect propofol pharmacokinetics. The present study included more women than men. Vuyk et al. [17] found that sex affects propofol pharmacokinetics at lower concentrations in female elderly patients than in male elderly patients. The age of our patients ranged from 30 years to 70 years. We found comparable sex-related differences in propofol concentrations. However, no significant difference was observed.

Vascular pain on injection is one disadvantage of propofol. Eriksson et al. [18] reported that $\mathrm{pH}$ changes on the vascular endothelium may modify propofol-induced pain on injection and that lignocaine mixed with propofol causes less pain. None of our patients experienced vascular pain; however, we may have injected a smaller bolus for induction than that used in the Marsh model. If so, the $\mathrm{pH}$ may not have affected the vascular endothelium as much as it does in the Marsh model.

Hypotension after propofol injection is also a concern. Wang et al. [19] found that the mean arterial blood pressure decreased by approximately $18 \%$ when achieving an effect-site concentration of $3.0 \mu \mathrm{g} / \mathrm{mL}$ by using a modified Marsh model. We believe that our model achieved a similar performance.

The findings of our study must be evaluated in consideration of some limitations. First, our sampling schedule did not include data for the time after the termination of propofol infusion. These data may be needed for building an exact PK model. Potential complications may be over dose. When the patient's metabolism and excretion are different from usual, propofol administration used by our model may be potency of overdose administration. However, the purpose of this article was to create a new model suitable for Japanese individuals, and to determine how to introduce and maintain anesthesia suitably for Japanese individuals. To that end, we believe that a stable blood concentration is an important serious matter during the introduction and maintenance periods. We believe that the change in the blood concentration from the dosage cancellation depends on the metabolic pathway of an individual, even if we use any models. Therefore, it was not the principal objective in this study. We also believe it can be substituted in the elapsed time to consciousness, which is a factor of pharmacodynamics but not $\mathrm{PK}$.

We could not calculate the pharmacodynamic parameters of propofol because 
general anesthesia was induced using fentanyl. In addition, fentanyl may affect vascular pain. We postulate that the effect of fentanyl would be small because propofol was injected immediately after the infusion of fentanyl. In addition, we did not have a sufficient number of blood samples to apply our model clinically. More samples are needed to increase the accuracy of propofol pharmacokinetic parameters.

\section{Conclusion}

In conclusion, we demonstrated that the General Purpose model, Schnider model, and Marsh model cannot be directly applied to Japanese patients who are infused with a constant rate of propofol. We created a new pharmacokinetic model that performed better than the other three models for Japanese populations.

\section{Acknowledgements}

We thank Shunsuke Hayashi for measuring the blood propofol concentrations.

\section{Conflicts of Interest}

The authors declare no conflicts of interest regarding the publication of this paper.

\section{References}

[1] Rush, E.C., Freitas, I. and Plank, L.D. (2009) Body Size, Body Composition and Fat Distribution: Comparative Analysis of European, Maori, Pacific Island and Asian Indian adults. British Journal of Nutrition, 102, 632-641.

[2] Jensen, B., Moritoyo, T., Kaufer-Horwitz, M., Peine, S., Norman, K., Maisch, M., Matsumoto, A., Masui, Y., Velázquez-González, A., Domínguez-García, J., FonzEnríquez, E., Salgado-Moctezuma, S.G. and Bosy-Westphal, A. (2019) Ethnic Differences in Fat and Muscle Mass and Their Implication for Interpretation of Bioelectrical Impedance Vector Analysis. Applied Physiology, Nutrition, and Metabolism, 44, 619-626. https://doi.org/10.1139/apnm-2018-0276

[3] Ramamoorthy, A., Pacanowski, M.A., Bull, J. and Zhang, L. (2015) Racial/Ethnic Differences in Drug Disposition and Response: Review of Recently Approved Drugs. Clinical Pharmacology \& Therapeutics, 97, 263-273. https://doi.org/10.1002/cpt.61

[4] Tachibana, N., Niiyama, Y. and Yamakage, M. (2014) Evaluation of Bias in Predicted and Measured Propofol Concentrations during Target-Controlled Infusions in Obese Japanese Patients: An Open-Label Comparative Study. European Journal of Anaesthesiology, 31, 701-707. https://doi.org/10.1097/EJA.0000000000000154

[5] Lampotang, S., Lizdas, D.E., Derendorf, H., Gravenstein, N., Lok, B. and Quarles, J.P. (2016) Race-Specific Pharmacodynamic Model of Propofol-Induced Loss of Consciousness. The Journal of Clinical Pharmacology, 56, 1141-1150. https://doi.org/10.1002/jcph.716

[6] Germovsek, E., Kent, A., Metsvaht, T., Lutsar, I., Klein, N., Turner, M.A., Sharland, M., Nielsen, E.I., Heath, P.T. and Standing, J.F. (2016) Development and Evaluation of a Gentamicin Pharmacokinetic Model that Facilitates Opportunistic Gentamicin Therapeutic Drug Monitoring in Neonates and Infants. Antimicrobial Agents and 
Chemotherapy, 60, 4869-4877. https://doi.org/10.1128/AAC.00577-16

[7] Yeganeh, M.H. and Ramzan, I. (1997) Determination of Propofol in Rat Whole Blood and Plasma by High-Performance Liquid Chromatography. Journal of Chromatography B: Biomedical Sciences and Applications, 691, 478-482. https://doi.org/10.1016/S0378-4347(96)00469-0

[8] Marsh, B., White, M. and Morton, N. (1991) Pharmacokinetic Model Driven Infusion of Propofol in Children. British Journal of Anaesthesia, 67, 41-48. https://doi.org/10.1093/bja/67.1.41

[9] Schnider, T.W., Minto, C.F. and Gambus, P.L. (1998) The Influence of Method of Administration and Covariates in the Pharmacokinetics of Propofol in Adult Volunteers. Anesthesiology, 88, 1170-1182. https://doi.org/10.1097/00000542-199805000-00006

[10] Eleveld, D.J., Proost, J.H., Cortínez, L.I., Absalom, A.R. and Struys, M.M. (2014) A General Purpose Pharmacokinetic Model for Propofol. Anesthesia \& Analgesia, 118, 1221-1237. https://doi.org/10.1213/ANE.0000000000000165

[11] Japanese Ministry of Health, Labour and Welfare (2018) National Health and Nutrition Survey 2016.

http://www.mhlw.go.jp/bunya/kenkou/eiyou/dl/h28-houkoku.pdf

[12] Lee, Y.H., Choi, G.H., Jung, K.W., Choi, B.H., Bang, J.Y., Lee, E.K., Choi, B.M. and Noh, G.J. (2017) Predictive Performance of the Modified Marsh and Schnider Models for Propofol in Underweight Patients Undergoing General Anaesthesia Using Target-Controlled Infusion. British Journal of Anaesthesia, 118, 883-891. https://doi.org/10.1093/bja/aex102

[13] Masui, K., Upton, R.N. and Doufas, A.G. (2010) The Performance of Compartmental and Physiologically Based Recirculatory Pharmacokinetic Models for Propofol: A Comparison Using Bolus, Continuous, and Target-Controlled Infusion Data. Anesthesia \& Analgesia, 111, 368-379. https://doi.org/10.1213/ANE.0b013e3181bdcf5b

[14] Rigouzzo, A., Servin F. and Constant, I. (2010) Pharmacokinetic-Pharmacodynamic Modeling of Propofol in Children. Anesthesiology, 113, 343-352. https://doi.org/10.1097/ALN.0b013e3181e4f4ca

[15] Glen, J.B. and Servin, F. (2009) Evaluation of the Predictive Performance of Four Pharmacokinetic Models for Propofol. British Journal of Anaesthesia, 102, 626-632. https://doi.org/10.1093/bja/aep043

[16] Chi, X., Pan, J., Cai, J., Luo, G., Li, S., Yuan, D., Rui, J., Chen, W. and Hei, Z. (2018) Pharmacokinetic Analysis of Propofol Target-Controlled Infusion Models in Chinese Patients with Hepatic Insufficiency. Medical Science Monitor, 24, 6925-6933. https://doi.org/10.12659/MSM.910103

[17] Vuyk, J., Oostwouder, C.J. and Vletter, A.A. (2001) Gender Differences in the Pharmacokinetics of Propofol in Elderly Patients during and after Continuous Infusion. British Journal of Anaesthesia, 86, 183-188. https://doi.org/10.1093/bja/86.2.183

[18] Eriksson, M., Englesson, S., Niklasson, F. and Hartvig, P. (1997) Effect of Lignocaine and pH on Propofol-Induced Pain. British Journal of Anaesthesia, 78, 502-506. https://doi.org/10.1093/bja/78.5.502

[19] Wang, H., Kuo, T.B., Chan, S.H., Tsai, T.H., Lee, T.Y. and Lui, P.W. (1996) Spectral Analysis of Arterial Pressure Variability during Induction of Propofol Anesthesia. Anesthesia \& Analgesia, 82, 914-919. https://doi.org/10.1097/00000539-199605000-00004 